\title{
Use of Cholesterol-Lowering Medications of Patients with Myocardial Infarction from 2007-2015 in Cardiology Clinic to "Mother Teresa" University Hospital in Albania
}

\author{
Kleva Shpati', Rudina Prifti' ${ }^{1}$, Dritan Shpati², Zana Bruci³, Erina Hilaj ${ }^{1}$ \\ ${ }^{1}$ Department of Pharmacy, Albanian University, Tirana, Albania \\ ${ }^{2}$ Hospital Mbreteresha Geraldine, Tirana University, Tirana, Albania \\ ${ }^{3}$ Faculty of Medicine, Tirana University, Tirana, Albania \\ Email: klevashpati@yahoo.com
}

Received 29 September 2015; accepted 26 June 2016; published 29 June 2016

Copyright @ 2016 by authors and Scientific Research Publishing Inc.

This work is licensed under the Creative Commons Attribution International License (CC BY). http://creativecommons.org/licenses/by/4.0/

\section{Open Access}

\section{Abstract}

Background: Coronary Heart Disease (CHD) remains the leading cause of morbidity and mortality in Albania. According to Institute of Statistics of Albania (ISA), CHD remains in the 5 first places caused mortality and morbidity in our country. Currently, all the protocol treatments to Mother Teresa University Hospital (MTUH) are focused on low-density lipoprotein cholesterol (LDL-C) as the primary target for risk reduction therapy, followed by triglycerides (TG) and high-density lipoprotein cholesterol (HDL). Our clinicians recommend that the intensity and target goals of LDL-C-lowering therapy should be adjusted to individual treatment. The choice of a specific drug from anti hypercholesterolemia class depends on several factors which need to be evaluated better from our health providers. Methods and Findings: Data was collected between 2007 to 2015 at Mother Teresa University Hospital (MTUH), Intensive Care Unit in Cardiology Clinic. 700 patients were treated with statins as follows: Fluvastain $40 \mathrm{mg}$ and $80 \mathrm{mg}$, Atorvastatin $10 \mathrm{mg}$ and $20 \mathrm{mg}$, Simvastatin and Rosuvastatin $20 \mathrm{mg}$. Statins are increased significantly during the years compared with fibrates. Their use grew from $87 \%$ of all statin types to $95 \%$ in 2015 , with different types from fluvastatin in 2007 to atorvastatin being the leading medication in 2015. Coronary Heart Disease, especially Myocardium Infarct, were studied as diseases when meanly the hypolipidic drugs were usually used in medical protocol treatment. Statins and fibrates were the therapy target to be studied when statin dominated the patients' treatment and fibrates were used in limited quantity. Atorvastatin is now dominating the market, while fluvastatin was on the first statin therapy for several years in the past. Fibrate were used in the modest quantities in value from $2 \%$ to $1.5 \%$ in years 2007 to 2015 . The use of statins in moderate-risk and high-risk patients showed continued growth subsequently, but stable with dosage of atorvastatin $20 \mathrm{mg}$. Lower 
statin dosage used was independently associated for all patients, young patients had used high dosage of statins and fluvastatin respectively $40 \mathrm{mg}$ and $80 \mathrm{mg}$. The missing high dosage of atorvastatin was notable. The values of LDL were improved but not in the level of efficacy of treatment. Conclusion: Statin therapy becomes more selected compared with fibrate. Atorvastatin $20 \mathrm{mg}$ remains the most important stain used in our clinic, but low dosage remains still a problem. The expected level of LDL-C is the target for the practitioners. More aggressive statins such as rosuvastatin start to be present in our clinic, but it is less used by the patients. Meanwhile the lifestyle, exercises, balance diet, smoking cessation are parts of the patients' files. The side effects are minors and the medication process has continued.

\section{Keywords}

\section{Statins, Fibrate, Coronary Heart Disease, Risk Factors, Adverse Drug Events, Etc.}

\section{Introduction}

Coronary Heart Disease (CHD) remains the leading cause of morbidity and mortality in Albania. According to Institute of Statistics of Albania (ISA), CHD remains in the 5 first places caused mortality and morbidity in our country. CHD is a group of disease that includes: stable angina, unstable angina, myocardial infarctions. It is within group of cardiovascular diseases and is the most common type. We have chosen this group for another additional reason: lipid-lowering products that are commonly used in practice as a part of protocol treatment. In 2013 CHD was the most common cause of death globally resulting 8.14 million deaths (16.8\%) up from 5.74 million deaths (12\%) in 1990 [1]. The effectiveness of lipid-lowering therapy in reducing the risk of cardiovascular morbidity and mortality has been firmly established in many clinical trials [2]-[6], and lipid-lowering drugs (LLDs) are frequently used in everyday practice. Our study is evaluated at Intensive Care Clinic of Mother Teresa University Hospital Tirana-Albania, where the LLDs drugs are used frequently. In recent years, the use of LLDs, especially 3-hydroxy-3-methylglutaryl coenzyme A (HMG-CoA) reductase inhibitors (statins), has rapidly increased according to Health Care Insurance Fond of Albania (HCIF) [6]. Risk factors such as smoking, diabetes, lack of exercises, obesity, poor diet and excessive alcohol were studied for achieving the goal of therapy. Currently, evidence-based practice focuses on low-density lipoprotein cholesterol (LDL-C) as the primary target for risk reduction therapy, Triglycerides (TG) and the High-Density Lipoprotein (HDL) were part of blood analysis test but they were sporadic and not continuously. The clinicians recommend that the intensity and target goals of LDL-C-lowering therapy should be the priority, in spite of having two other analysis results such as TG and HDL that may help them in a better treatment conclusion. CHD risk is categorized as low, moderate, or high based on the presence or absence of CHD.

The patients who had high and moderate risk has shown that LDL-C level and the statin therapy was inadequate due to the low dosage used in many report cases. Among existing drug therapies, 3-hydroxy-3-methyl-glutaryl coenzyme A reductase inhibitors, more commonly known as statins, has enter only last ten years in Albania. Statins are presented in Albanian market. First statin was simvastatin registered at 2001, one year later it had been reimbursed form Insurance Health Care Institute. The other types of statins such as fluvastatin, atorvastatin and pravastatin are registered later and reimbursed only fluvastatin $40 \mathrm{mg}$ and $80 \mathrm{mg}$ has dominated the Albanian market for many years, when finger has been changed in the atorvastain $20 \mathrm{mg}$ favor [2] [5]. Fibrates were registered in 2000, but their use have had not a significance consume compared with statins in the other countries [3] [6]-[8]. The treatment is a complex phenomenon in which the physician, the patient, and the presence of various co-morbidities all play a role. As the lipid-altering mechanism, potency and safety profile of various LLDs are different [6] [7] [9], and the choice of a specific drug may depend on the type of hyperlipidemia as well as on pre-existing co-morbidities. Statin is studied for adverse events as well. The most important adverse effects associated with statins (atorvastatin, fluvastatin, lovastatin, pravastatin, rosuvastatin, and simvastatin) are asymptomnts which are collected in our study. The biochemistry data show increases in liver transaminases and myopathy. Therefore, low dosage of stains used was a relevant factor in determining the safety profile of statins. Pharmacological differences are evident among the statins, and these may affect their 
safety and potential for drug interactions. The withdrawal of cerivastatin in August 2001 because of its association with fatal rhabdomyolysis underscores the importance of considering the safety profile of the statins [10] [11].

\section{Materials and Methods}

Data from 2007 through 2015 were obtained from the patient files archived to Statistic Department to Mother Teresa University Hospital (MTUH). 780 patients were treated with statins. Statin used were six types of them, atorvastatin, lovastatin, pravastatin, simvastatin, fluvastatin, rosuvastatin: Serum total cholesterol, LDL-Cholesterol, HDL-cholesterol and triglyceride levels were determined before and after hospitalization. Meanwhile the patients have been followed six months later with the same treatment according to hospital protocol. The Department for Health Statistics provides complete descriptions of statins used, dosage, main cause of hospitalization, co-morbidement, biochemistry data included and lipidgram chart etc. Initiated lipid-lowering therapy in clinic was mainly by prescribing a statin (atorvastatin, fluvastatin, pravastatin, simvastatin and rosuvastatin), followed by a fibrate (ciprofibrate, and fenofibrate), but in very limited prescribers. We collected the following data on the baseline characteristics to evaluate the effects of potential determinants on prescribing LLDs: demographic and patient characteristics age and gender, number of risk factors for CHD (other than serum cholesterol, hospitalization, and diagnosis of myocardial infarction (MI) or stroke in the prior 6 months; We estimated CHD risk for adults aged $20 \mathrm{y}$ and older based on risk factor counting. CHD risk was mutually exclusively categorized as low ( $0-1$ risk factors), moderate ( $2+$ risk factors), or high (CHD, other atherosclerotic diseases, or diabetes) The moderate-risk group included visits by patients without CHD or equivalent but with at least two of the following risk factors: age (for men, $>45 \mathrm{y}$; for women, $>55 \mathrm{y}$ ), cigarette smoking, or a physician-reported diagnosis of hypertension. Unfortunately, the other risk factors-family history of premature, body index weight, were not collected due to limited data. Data source provided actual cholesterol measurements. Disease conditions were identified by International Classification of Disease (ICD-9) codes [3]. For instance, we identified patients as having dislipidemia, diabetes Mellitus type II, arterial fibrillation etc. The characteristics included patient age, gender were used in the function of treatment showing efficacy.

In our study we have used ATC/DDD methodology known by the World Health Organization [4] as a standard for drugs use. We have used this methodology as an official method for Albanian Authorities, Hospitals and HCIF. This drugs are included in the category C10 respectively C10AA hypolipidic drugs and forth level were included he inhibitors of HMG-Coa or well known as statins, C10AB are fibrates [5]. Data from 2007 through 2015, tracked trends in statin use in Albania during hospitalization patients and control of lipids levels for 6 months after hospitalization according to the recommendation of the Fond of Insurance of Healthcare for Hypercholesterolemia control issued by and protocol treatment. In addition, we analyzed the characteristics with statin use for insights as to how to target interventions to improve statin use Statin used was the first goal and the relationship of statin used to patient characteristics such as age and gender, statins used were: atorvastatin, lovastatin, pravastatin, simvastatin, fluvastatin, rosuvastatin. Statistical analyses were performed using SPSS versions 15. The independent effect of each patient visit characteristic on statin use after controlling for all other characteristics was assessed with multivariate logistic regression.

\section{Results}

Statins were used among patients with Myocardial Infarction (differ types IM) Statins used for IM were 87\% of in 2007 and 95\% in 2015. The dominance of statins as lipid-lowering agents grew markedly of all lipid-lowering medications compare with fibrate which has a very lower linebase from 6\% to 3\% in 2007 to 2015 in Albania. The study comprised 780 patients, including 680 patients (2007) and 741 (2015) with LLDs statin users, 46 patient (2007) and 23 patient (2015) fibrates. Patients without lipid lowering drugs, were very limited in number 54 patient in 2007 and 16 in 2015 (Table 1). A diagnosis of CHD particularly MI (all types) was the most common reason for initiating LLD therapy, followed by a diagnosis of hyperlipidemia and other atherosclerotic diseases, diabetes mellitus type II, etc.

Among available statins, fluvastatin remained the therapeutic choice for 2007, after which it was surpassed by atorvastatin, rosuvastatin and simvastatin. Atorvastatin constituted 59.5\% (26\% - 64\%) fluvastatin 26\% (61\% $12 \%$ ) of all statin use in 2015. Rosuvastatin has started to become a used statin after atorvastatin in three last 
Table 1. The LLDs used to mother teresa hospital cardiology clinic during the period 2007-2015 (statins and fibrates) (III).

\begin{tabular}{cccc}
\hline Number of patients with & $\begin{array}{c}\text { Number of patients with } \\
\text { lipid-lowering drugs statin } \\
(\%)(\mathrm{n}=680)\end{array}$ & $\begin{array}{c}\text { Number of patients without } \\
\text { lipid-lowering drugs fibrate } \\
(\mathrm{n}=46)\end{array}$ & $\begin{array}{c}\text { lowering drugs } \\
(\mathrm{n}=54)\end{array}$ \\
2007 & $67(\%)$ & $6(\%)$ & Number of patients without \\
2015 & $\begin{array}{c}\text { Number of patients with } \\
\text { lipid-lowering drugs statin } \\
(\%)(\mathrm{n}=741)\end{array}$ & $\begin{array}{c}\text { Number of patients with } \\
\text { lipid-lowering drugs fibrate } \\
(\%)(\mathrm{n}=16)\end{array}$ & $2(\%)$ \\
\hline
\end{tabular}

year of the study. High risk patient resulted in greater statin use, and the divergence in statin use among the three risk categories has not grown in recent years. Missing classification in the files statin dosage used for this purposes create problems on efficacy of treatment. Age and gender give us interesting results for statin used. We estimated CHD risk for adults aged 20 y and older based on risk factor counting. CHD risk was mutually exclusively categorized as low ( $0-1$ risk factors), moderate $(2+$ risk factors), or high (CHD, other atherosclerotic diseases, or diabetes). The moderate-risk group included visits by patients without CHD or equivalent but with at least two of the following risk factors: age (for men, $>45 \mathrm{y}$; for women, $>55 \mathrm{y}$ ), cigarette smoking, or a physician-reported diagnosis of hypertension. The most risqué group are three from 60 - 70 (y) with 275 patients 275 , 50 - 60 (y) with 206 patients and 70 - 80 (y) with 146 patients. Detailed data are given in the Table 2.

All the data collected for the period 2007-2014 the average age was as per Table 3. The average was 64.59 that it is younger comer with previous years according to the Statistical department of MTUH and.

The most risked population by gender is men compare with women. The data shows us that the men have a range of disease increased compare with women. This fact has been demonstrated with Graphic 1 which interpreted clearly such as result.

The severity of cardiovascular diseases and diabetes was also associated with treatment initiation of LLDs. LLD therapy consistently increased with disease severity. Such a trend remained for CHD, CHF, diabetes, and peripheral vascular disease, after adjusting for demographic and patient characteristics (test for trend with $P=$ 0.0001). Means that high risk patients were significantly compare with moderate risk patients.

As expected, treatment initiation of LLDs was also associated with serum lipid concentrations (Table 4). LDL measure was target biochemistry analyses for all the patients. The results received were good and the patient has reached such a result after six months treatment according to the protocol treatment of Hospital and Insurance Institute. After this period the patients are oblige to evaluate the test analyses for lipids results. In Graphic 2 shows the laboratory results for LDL of all patients treated with statins.

These results are showed by the Graphic 2.

The most important adverse effects associated with statins (atorvastatin, fluvastatin, lovastatin, pravastatin, rosuvastatin, and simvastatin) are asymptomatic increases in liver transaminases and myopathy. Based on this clinical evidence, the U.S. National Cholesterol Education Program (NCEP) Adult Treatment Panel (ATP) III report extended the use of lipid-lowering treatments to a larger number of high-risk CHD patients who often receive more than one medication. During our study we have observed adverse drug events due to the co-commitment with other medicines and during the treatment. Such a symptoms of gastrointestinal one placed in $50 \%$ of the cases, fatigue $12 \%$, headache $16 \%$, rash $2 \%$.

\section{Discussion}

Statins are very potent inhibitors of HMG-CoA reductase. Two decades passed since the first publications, the 4S study [7] establishes the usefulness of statin therapy in post AMI patients. However, there still exist a numerous scientific publications demonstrating the beneficial effects of statis and the low rate of implementing the guideline in practice. While many studies were conducted with statin therapy such as LIPID [8], ASCOT-LLA [9] CARDS [12] PROVE IT: TIMI 22 [13] TNT CKD) [14], IDEAL [15], REVERSAL [16], as well Heart Protection Study [17] with doses 20 - $40 \mathrm{mg} /$ day where the initiating dose is $10 \mathrm{mg} / \mathrm{day}$

Recently, it has even been suggested in some well controlled studies such as NCEP for LDL-C goal need to be implemented in our protocol treatments in Albania. These data are focused on LDL-C levels reaching 70 - 80 $\mathrm{mg} / \mathrm{dl}$. The current NCEP guideline discuss LDL goals the routine dose of satin use above $40 \mathrm{mg} / \mathrm{daily}$ for post 
Table 2. The patients' age for the period 2007-2015 with CVD classified with group ages (10 y).

\begin{tabular}{cccc}
\hline & Age (years) & $\begin{array}{c}\text { Number of patients with MI (2007-2015) } \\
\text { (moderate risk patients) }\end{array}$ & $\begin{array}{c}\text { Number of patients with MI (2007-2015) } \\
\text { (high risk patients) }\end{array}$ \\
\hline 1 & $<30$ & 2 & 0 \\
2 & $30-40$ & 10 & 6 \\
3 & $40-50$ & 31 & 8 \\
4 & $50-60$ & 206 & 10 \\
5 & $60-70$ & 275 & 2 \\
6 & $70-80$ & 146 & 2 \\
7 & $>80$ & 82 & 0 \\
\hline
\end{tabular}

Table 3. Average age for the patients studied during the period 2007-2015.

\begin{tabular}{cc}
\hline Statisticals data & \\
\hline Patients (n) & 780 \\
Average & 64.59 \\
Standard deviation & 11.14 \\
Minimum $^{*}$ & 15.00 \\
Maximum & 93.00 \\
\hline
\end{tabular}

Table 4. Laboratory results with LDL-C.

\begin{tabular}{cccc}
\hline & Average \pm 2 standard deviations & Significance (p) & Results \\
\hline LDL Entry LDL post treatment & $226.2714 \pm 78.13248177 .1476 \pm 71.76067$ & $\mathrm{p}=0.002$ & $-38 \%$ \\
\hline
\end{tabular}

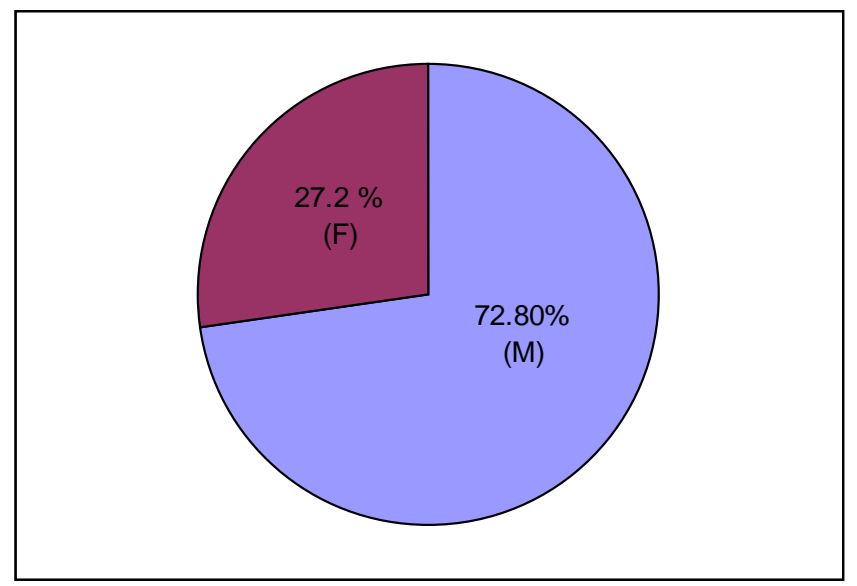

Graphic 1. The patients’ gender for the period 2007-2015 with CVD.

IM patients [17]-[20].

Despite of increases from 2007 to 2015 the use of statins, the magnitude of improving results of LDL is smaller than expected and the rate of use remains suboptimal according to the best available evidence This data are shown in Graphic 3. When evaluating statin use across different CHD risk categories, the observed trends raise several issues. Both the rate of statin use and the absolute increases in the rate over time were positively associated with the level of CHD risk, which appropriately conforms to the notion of risk stratification. The associations 


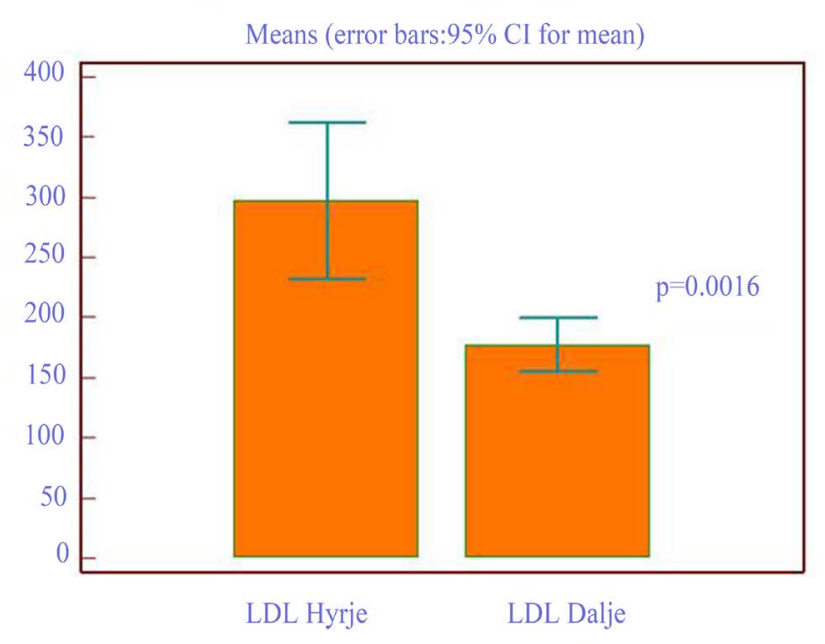

Graphic 2. Laboratory results for LDL before and after hospitalization for the period 2007-2015.

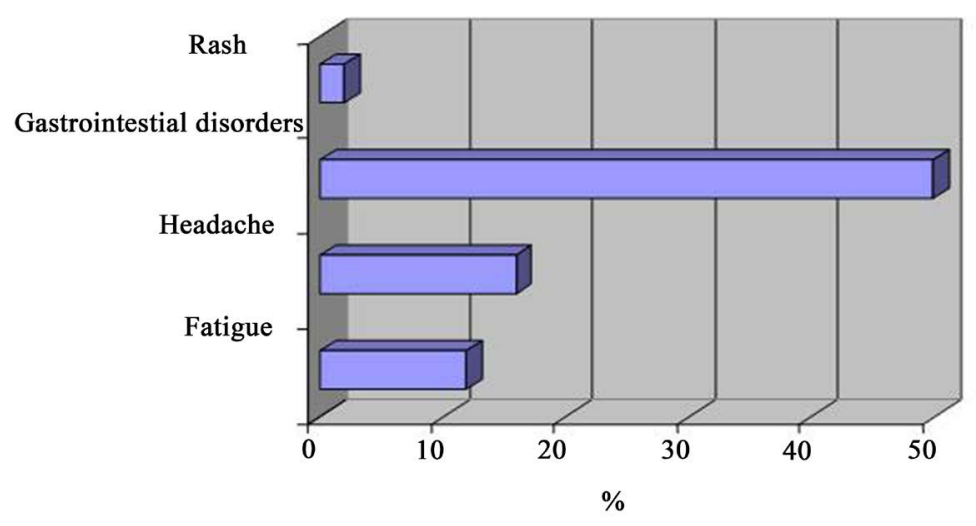

\section{Graphic 3. Adverse drug evenst (ADE).}

persisted after adjusting for potentially confounding factors such as a age and gender as well other diagnosis and nonclinical patient visit characteristics [21] [22]. As it is shown in the Table 2 when the age was the first factor studied two group ages from 50 - 60 years and 60 - 70 years where he most risk population. According to the global evidences [23]-[27] the age is younger and the population with cardiovascular disease in Albania it seems a problematic issues when few factors may influence such a missing primary care systems and uncontrolled patients periodically. The average of the age is 64.59 years which can load a lot of social problems.

In 2002, one year after the publication of Adult Treatment Panel III [3], statins were reportedly used in only $19 \%$ of patient visits with established CHD or its equivalents, and the average rate was no higher than $50 \%$ among high-risk visits where a diagnosis of hyper lipidemia also was noted. These data suggest a dramatic treatment gap. Another analysis based on national data estimated that $72 \%$ of Americans with existing CHD would benefit from drug therapy to achieve the target LDL-C goal of $2.59 \mathrm{mmol} / \mathrm{l}(100 \mathrm{mg} / \mathrm{dl})$ or less, assuming a $10 \%$ LDL-C reduction with diet [17]. However, only $11 \%$ of those eligible individuals received lipid-lowering drug therapy, suggesting a gap of $89 \%$ [28]. These obvious treatment gaps are disconcerting; especially in light of the recent Adult Treatment Panel III update [3] that supports more intensive lipid-lowering drug therapy for patients at high and moderately high risk for a heart attack. Barriers to adequate treatment of high-risk patients may stem from the patient (e.g., lack of drug adherence, concern about adverse effects, inadequate knowledge of their hyper lipidemia, and drug cost), the physician (e.g., lack of guideline awareness, failure to measure lipid levels, and overestimation of actual treatment), and the health-care system (e.g., lack of monitoring and followup and emphasis on acute medical problems) [24] [29]-[33]. 
Statins are presented in Albanian market. First statin was simvastatin registered at 2001, one year later has been reimbursed form HCIF in the years when Adult Treatment Panel III was in powr for many developed country. Having officially by registered medicines the statins, in Albania market it doesn't mean to respect what ATP III recommended. The other types of statins such as fluvastatin, atorvastatin and pravastatin are register later and reimbursed to upon their registration mostly from the influence of pharmaceutical company than the clinical needs. Fluvastatin $40 \mathrm{mg}$ and $80 \mathrm{mg}$ has dominated the Albanian market for many years, when finger has been changed in the atorvastain $20 \mathrm{mg}$ favor [3] [5]. Fibrate were register in 2000, but their use has had not a significance consume of use compare with statins in the other study's countries. They were quite stable and not with evidences explained their use [34]-[38].

Our data shows an increase in statin use from 87\% of high-risk patient in 2007 to 95\% in 2015, but without continued growth subsequently 5 .

National Health and Nutrition Examination Survey III data showed that $60 \%$ of 38.5 million adult Americans without CHD who had two or more risk factors had an LDL-C level above the recommended $3.36 \mathrm{mmol} / \mathrm{l}$ (130 $\mathrm{mg} / \mathrm{dl}$ ) and that $45 \%$ would remain eligible for drug therapy even after a $10 \%$ decrease in LDL-C with diet. In addition, Fedder et al. [24] found a doubling effect in the number eligible for primary prevention drug therapy by switching to Framingham risk scoring [20]. Other researchers have reported that the proportions of treatment-eligible primary prevention patients who received no drug therapy reached as high as 97\% [39]-[41].

In our study, statin use wasn't reported patients with diseases stories such as hyperlipidemia (unknown) or diabetes mellitus II. Most of the patients (97\% of them) start to use stain for the first time in spite of necessary treatment with them for primary prevention. The patients with post IM the treatments was stopped after a period of statin use. For instance, a survey conducted in nine European countries found that only 32\% of patients with confirmed CHD received lipid-lowering medications [42]. Likewise, in a population-based study from the Netherlands, merely $16 \%$ of individuals eligible for lipid-lowering drugs were actually treated [7]. In our country only $3 \%$ of the patients confirmed use of stains in the post IM status.

In spite of being clearly underused, statins increasingly dominate lipid-lowering drug therapy, accounting for $87 \%$ of all lipid-lowering medications used in 2007 to $95 \%$ in 2015, which confirms the trends increased in Albania [3].

Also, in concert with other researchers [23], we observed a shift in the leading statin prescribed, from fluvastatin to atorvastatin, corresponding to the market entry. Atorvastatin accounted for over half of all statin use in 2008 (at 2007 fluvastatin was 61\%). Even though most statins share similar tolerability, some evidence shows that atorvastatin has greater dose-specific potency for lowering LDL-C [24].The publication of the Scandinavian Simvastatin Survival Study in 1994 [37], and two other statin trials during recent years [16] [41], could have affected the initial choice of LLDs as well. As there are no clinically appreciable differences in the safety profiles of these two statins for the 'significant' determinants were modest, the differences could have arisen by chance, or through unmeasured baseline characteristics. In Albania the trend of statin type used is different compare with 4S study. In spite of entry later on the market. The market is shared with atorvastatine and fluvastatin, completely different from simvastatin and pravastatin which are present on the market but not with significat fingers. Today atorvastatin is dominating the market.

Our findings must be interpreted in the context of data limitations. Also, we haven't classifications by risk factors who have not been seen by a physician or whose risk factors failed to be recorded. Lack of detailed clinical data prohibits accurate risk assessment based on Framingham risk scoring. While the risk factor counting statin use in relation to the latest lipid-lowering guidelines, but unfortunately this terms of evaluations are not according to good clinical practice protocol. We likely misclassified some patients as moderate risk when they may have actually been high risk despite the absence of CHD or CHD equivalents. On the other hand, indications for statins might be marginal for some young patients with modestly elevated risk factors.

\section{Conclusions}

The use of statin therapy was increased from 2007-2015, atovastatin remained the first line treatment to Mother Teresa University Hospital in Albania. The group age with higher risk for CHV (IM) is group 50 - 60 y and 60 $70 \mathrm{y}$, men are more risked compared with female with $72.8 \%$.

Guideline for cardiovascular risk reduction needs to be implemented accordingly in our clinic, where patients can benefit from statin therapy. Given the fact that observed practice shortfalls, drug therapy in moderate-risk 
and high-risk patients remains a top priority for the improvement in our clinic.

The dosage of statin should be used according to the patients' needs. The side effects are minor and the medication process has continued normally. They are under control from the healthcare providers.

\section{References}

[1] GBD 2013 Mortality and Causes of Death, Collaborators (2014) Global, Regional, and National Age-Sex Specific AllCause and Cause-Specific Mortality for 240 Causes of Death, 1990-2013: A Systematic Analysis for the Global Burden of Disease Study 2013. Lancet, 385, 117-171.

[2] National Cholesterol Education Program (NCEP) Expert Panel on Detection, Evaluation, and Treatment of High Blood Cholesterol in Adults (Adult Treatment Panel III) (2002) Third Report of the National Cholesterol Education Program (NCEP): Final Report. Circulation, 106, 3143-3421.

[3] Albanian Institute of Statistics (INSTAT). http://www.instat.gov.al/al/home.aspx

[4] Who Collaboring Centre for Drug Statistics. Methodology ATC Index with DDDs. (2009) WHOCC-ATC/DDD Index. WHO, Oslo. www.whocc.no/atc_ddd_index/

[5] American Heart Association (2002) Heart Disease and Stroke Statistics—2005 Update. American Heart Association, Dallas.

[6] Health Care Insurance Fond of Albania—Fondi i Sigurimeve te Kujdesit Shendetesor.

[7] Expert Panel on Detection, Evaluation, and Treatment of High Blood Cholesterol in Adults (2001) Executive Summary of the Third Report of the National Cholesterol Education Program (NCEP) Expert Panel on Detection, Evaluation, and Treatment of High Blood Cholesterol in Adults (Adult Treatment Panel III). JAMA, 285, 2486-2497. http://dx.doi.org/10.1001/jama.285.19.2486

[8] Scandinavian Simvastatin Survival Study Group (1994) Randomized Trial of Cholesterol Lowering in 4444 Patients with Coronary Heart Disease: The Scandinavian Simvastatin Survival Study (4S). Lancet, 344, 1383-1389.

[9] The LIPID Study Group (2002) Long-Term Effectiveness and Safety of Pravastatin in 9014 Patients with Coronary Heart Disease and average Cholesterol Concentrations: The LIPID Trial Follow-Up. Lancet, 359, 1379-1387. http://dx.doi.org/10.1016/S0140-6736(02)08351-4

[10] Brown, B.G., Zhao, X.Q., Sacco, D.E. and Albers, J.J. (1993) Lipid Lowering and Plaque Regression. New Insights into Prevention of Plaque Disruption and Clinical Events in Coronary Disease. Circulation, 87, 1781-1791. http://dx.doi.org/10.1161/01.CIR.87.6.1781

[11] Fattinger, K., Roos, M., Vergeres, P., Holenstein, C., Kind, B., Masche, U., et al. (2000) Epidemiology of Drug Exposure and Drug Adverse Reactions in Two Swiss Departments of Internal Medicine. British Journal of Clinical Pharmacology, 49, 158-167. http://dx.doi.org/10.1046/j.1365-2125.2000.00132.x

[12] Sever, P.S., et al. (2003) Prevention of Coronary and Stroke Events with Atorvastatin in Hypertensive Patients who Have Average or Lower-Than-Average Cholesterol Concentrations, in the Anglo-Scandinavian Cardiac Outcomes Trial—Lipid Lowering Arm (ASCOT-LLA): A Multicentre Randomised Controlled Trial. Lancet, 361, 1149-1158. http://dx.doi.org/10.1016/S0140-6736(03)12948-0

[13] Colhoun, H., et al. (2004) Primary Prevention of Cardiovascular Disease with Atorvastatin in Type 2 Diabetes in the Collaborative Atorvastatin Diabetes Study (CARDS): Multicentre Randomised Placebo-Controlled Trial. Lancet, 364, 685-696. http://dx.doi.org/10.1016/S0140-6736(04)16895-5

[14] Cannon, C.P., et al. (2004) Intensive versus Moderate Lipid Lowering with Statins after Acute Coronary Syndromes. The New England Journal of Medicine, 350, 1495-1504. http://dx.doi.org/10.1056/NEJMoa040583

[15] Shepherd, J., et al. (2006) Effect of Lowering LDL Cholesterol Substantially Below Currently Recommended Levels in Patients With Coronary Heart Disease and Diabetes. Diabetes Care, 29, 1220-1226. http://dx.doi.org/10.2337/dc05-2465

[16] Shepherd, J., et al. (2008) The Treating to New Targets Chronic Kidney Disease Sub-Study (TNT CKD). Journal of the American College of Cardiology, 51, 1448-1554. http://dx.doi.org/10.1016/j.jacc.2007.11.072

[17] Pedersen, T.R., et al. (2005) The Incremental Decrease in End Points through Aggressive Lipid Lowering Study (IDEAL). JAMA, 294, 2437-2424. http://dx.doi.org/10.1001/jama.294.19.2437

[18] Nissen, S.E. et al. (2004) Effect of Intensive Compared With Moderate Lipid-Lowering Therapy on Progression of Coronary Atherosclerosis. JAMA, 291, 1071-1080. http://dx.doi.org/10.1001/jama.291.9.1071

[19] Euro Med Stat Project (2004) The Library of European Union Pharmaceutical Indicators.

[20] The Norwagian Social Insurance Institution (2010) Finnish Statistics on Medicines. 
[21] Teeeling, M., Bennett, K. and Feely, J. (2005) The Influence of Guidelines on the Use of Statins: Analysis of Prescribing Trends 1998-2002. British Journal of Clinical Pharmacology, 59, 227-232. http://dx.doi.org/10.1111/j.1365-2125.2004.02256.x

[22] Avorn, J., Monette, J., Lacour, A., et al. (1998) Persistence of Use of Lipid-Lowering Medications: A Cross-National Study. JAMA, 279, 1458-1462. http://dx.doi.org/10.1001/jama.279.18.1458

[23] The EURO-MED-STAT Group (2003) Monitoring Expenditure and Utilisation of Medicinal Products in the European Union Countries: A Public Health Approach. The European Journal of Public Health, 13, 95-100. http://dx.doi.org/10.1093/eurpub/13.suppl_3.95

[24] Jacobson, T.A., Griffiths, G.G., Varas, C., Gause, D., Sung, J.C., et al. (2000) Impact of Evidence-Based "Clinical Judgment” on the Number of American Adults Requiring Lipid-Lowering Therapy Based on Updated NHANES III Data. National Health and Nutrition Examination Survey, Archives of Internal Medicine, 160, 1361-1369. http://dx.doi.org/10.1001/archinte.160.9.1361

[25] Fedder, D.O., Koro, C.E. and L’Italien, G.J. (2002) New National Cholesterol Education Program III Guidelines for Primary Prevention Lipid-Lowering Drug Therapy: Projected Impact on the Size, Sex, and Age Distribution of the Treatment-Eligible Population. Circulation, 105, 152-156. http://dx.doi.org/10.1161/hc0202.101971

[26] Sacks, F.M., Pfeffer, M.A., Moye, L.A., et al. (1996) The Effect of Pravastatinon Coronary Events after Myocardial Infarction in Patients Withaverage Cholesterol Levels. The New England Journal of Medicine, 335, 1001-1009. http://dx.doi.org/10.1056/NEJM199610033351401

[27] Pearson, T.A., Laurora, I., Chu, H. and Kafonek, S. (2000) The Lipid Treatment Assessment Project (L-TAP): A Multicenter Survey to Evaluate the Percentages of Dyslipidemic Patients Receiving Lipid-Lowering Therapy and Achieving Low-Density Lipoprotein Cholesterol Goals. Archives of Internal Medicine, 160, 459-467. http://dx.doi.org/10.1001/archinte.160.4.459

[28] Olson, K.L., Bungard, T.J. and Tsuyuki, R.T. (2001) Cholesterol Risk Management: A Systematic Examination of the Gap from Evidence to Practice. Pharmacotherapy, 21, 807-817. http://dx.doi.org/10.1592/phco.21.9.807.34553

[29] Stafford, R.S., Blumenthal, D. and Pasternak, R.C. (1997) Variations in Cholesterol Management Practices of US Physicians. Journal of the American College of Cardiology 29, 139-146. http://dx.doi.org/10.1016/S0735-1097(96)00441-X

[30] Grundy, S.M., Cleeman, J.I., Merz, C.N., Brewer, H.B., Clark, L.T., et al. (2004) Implications of Recent Clinical Trials for the National Cholesterol Education Program Adult Treatment Panel III Guidelines. Circulation, 110, $227-239$. http://dx.doi.org/10.1161/01.CIR.0000133317.49796.0E

[31] Wang, T.J., Stafford, R.S., Ausiello, J.C. and Chaisson, C.E. (2001) Randomized Clinical Trials and Recent Patterns in the Use of Statins. American Heart Journal, 141, 957-963. http://dx.doi.org/10.1067/mhj.2001.115587

[32] Jones, P., Kafonek, S., Laurora, I. and Hunninghake, D. (1998) Comparative Dose Efficacy Study of Atorvastatin versus Simvastatin, Pravastatin, Lovastatin, and Fluvastatin in Patients with Hypercholesterolemia (The CURVES Study). American Journal of Cardiology, 81, 582-587. http://dx.doi.org/10.1016/S0002-9149(97)00965-X

[33] Ayanian, J.Z., Landon, B.E., Landrum, M.B., Grana, J.R. and McNeil, B.J. (2002) Use of Cholesterol-Lowering Therapy and Related Beliefs among Middle-Aged Adults after Myocardial Infarction. Journal of General Internal Medicine, 17, 95-102. http://dx.doi.org/10.1046/j.1525-1497.2002.10438.x

[34] Ayanian, J.Z., Landrum, M.B. and McNeil, B.J. (2002) Use of Cholesterol-Lowering Therapy by Elderly Adults after Myocardial Infarction. Archives of Internal Medicine, 162, 1013-1019. http://dx.doi.org/10.1001/archinte.162.9.1013

[35] Baxter, C., Jones, R. and Corr, L. (1998) Time Trend Analysis and Variations in Prescribing Lipid Lowering Drugs in General Practice. BMJ, 317, 1134-1135. http://dx.doi.org/10.1136/bmj.317.7166.1134

[36] Majeed, A., Moser, K. and Maxwell, R. (2000) Age, Sex and Practice Variations in the Use of Statins in General Practice in England and Wales. Journal of Public Health, 22, 275-279. http://dx.doi.org/10.1093/pubmed/22.3.275

[37] Pyorala, K., De Backer, G., Graham, I., Poole-Wilson, P. and Wood, D. (1994) Prevention of Coronary Heart Disease in Clinical Practice. Recommendations of the Task Force of the European Society of Cardiology, European Atherosclerosis Society and European Society of Hypertension. Atherosclerosis, 110, 121-161. http://dx.doi.org/10.1016/0021-9150(94)90200-3

[38] NHS Executive (1997) Standing Medical Advisory Committee on Use of Statins. Department of Health, London.

[39] LaRosa, J.C., He, J.V. and Upputuri, S. (1999) Effect of Statins on Risk of Coronary Disease: A Meta-Analysis of Randomized Controlled Trials. JAMA, 282, 2340-2346. http://dx.doi.org/10.1001/jama.282.24.2340

[40] Grundy, S.M., Cleeman, J.I., Rifkind, B.M. and Kuller, L.H. (1999) Cholesterol Lowering in the Elderly Population. For the Coordinating Committee of the National Cholesterol Education Program, Archives of Internal Medicine, 159, 1670-1678. http://dx.doi.org/10.1001/archinte.159.15.1670 
[41] Marciniak, T.A., Ellerbeck, E.F., Radford, M.J., et al. (1998) Improving the Quality of Care for Medicare Patients with Acute Myocardial Infarction: Results from the Cooperative Cardiovascular Project. JAMA, 279, 1351-1357. http://dx.doi.org/10.1001/jama.279.17.1351

[42] US General Accounting Office (1998) Medicare HMOs: Specialists Provide More Appropriate Drug Therapy for Heart Attack Survivors. US General Accounting Office, Washington DC.

\section{Submit or recommend next manuscript to SCIRP and we will provide best service for you:}

Accepting pre-submission inquiries through Email, Facebook, Linkedin, Twitter, etc A wide selection of journals (inclusive of 9 subjects, more than 200 journals)

Providing a 24-hour high-quality service

User-friendly online submission system

Fair and swift peer-review system

Efficient typesetting and proofreading procedure

Display of the result of downloads and visits, as well as the number of cited articles

Maximum dissemination of your research work

Submit your manuscript at: http://papersubmission.scirp.org/ 